\title{
Recreational Drug Use among Chinese Men Who Have Sex with Men: A Risky Combination with Unprotected Sex for Acquiring HIV Infection
}

\author{
Jun-Jie Xu, ${ }^{1,2}$ Han-Zhu Qian,, ${ }^{3,4}$ Zhen-Xing Chu, ${ }^{1,2}$ Jing Zhang, ${ }^{1,2}$ Qing-Hai Hu, ${ }^{1,2}$ \\ Yong-Jun Jiang, ${ }^{1,2}$ Wen-Qing Geng, ${ }^{1,2}$ Christiana Meng Zhang, ${ }^{5}$ and Hong Shang ${ }^{1,2}$ \\ ${ }^{1}$ Key Laboratory of AIDS Immunology of National Health and Family Planning Commission, Department of Laboratory Medicine, \\ The First Affiliated Hospital, China Medical University, Shenyang 110001, China \\ ${ }^{2}$ Collaborative Innovation Center for Diagnosis and Treatment of Infectious Diseases, Hangzhou, China \\ ${ }^{3}$ Vanderbilt Institute for Global Health, Vanderbilt University, Nashville, TN, USA \\ ${ }^{4}$ Division of Epidemiology, Department of Medicine, Vanderbilt University Medical Center, Nashville, TN, USA \\ ${ }^{5}$ Georgetown University School of Medicine, Washington, DC, USA
}

Correspondence should be addressed to Hong Shang; hongshang100@hotmail.com

Received 21 February 2014; Accepted 28 March 2014; Published 15 April 2014

Academic Editor: Josep M. Llibre

Copyright (C) 2014 Jun-Jie Xu et al. This is an open access article distributed under the Creative Commons Attribution License, which permits unrestricted use, distribution, and reproduction in any medium, provided the original work is properly cited.

Objective. To investigate the prevalence of recreational drug use and its relationship with HIV infection among Chinese MSM. Methods. A cross-sectional study of 625 MSM was conducted in Shenyang, China. Questionnaires were administered to collect information on recreational drug use and sexual behaviors. Blood specimens were collected to test for HIV and syphilis antibodies. Results. Nearly a quarter $(23.2 \%, 145 / 625)$ of participants reported ever using recreational drugs, among which alkyl nitrites (poppers) was the most frequently used drug (19.2\%), followed by methylmorphine phosphate (5.1\%), methamphetamine (4.0\%), and ketamine (0.8\%). The overall prevalence of HIV and syphilis was $9.6 \%$ and $10.4 \%$, respectively. Multivariate logistic analysis showed that recreational drug use was significantly correlated with age $\leq 25$ year (adjusted odds ratio [aOR] $=1.6,95 \% \mathrm{CI}, 1.1-2.9$ ), single marital status $(\mathrm{aOR}=2.1,95 \% \mathrm{CI}, 1.2-3.6)$, and seeking male sexual partners mainly through Internet $(\mathrm{aOR}=1.8,95 \% \mathrm{CI}$, 1.8-2.8). Recreational drug use was independently associated with an increased risk of HIV infection $(\mathrm{aOR}=3.5,95 \% \mathrm{CI}, 2.0-6.2)$. Conclusions. Our study suggests that recreational drug use is popular among Chinese MSM and is associated with significantly increased HIV infection risk. HIV prevention intervention programs should reduce both drug use and risky sexual behaviors in this population.

\section{Introduction}

Abuse of recreational drugs (also known as club drugs, new-type drugs) [1-3] among men who have sex with men (MSM) has been a worldwide public health concern $[1,2,4-$ 7]. Compared with traditional illicit drugs (e.g., heroin and marijuana, etc.), recreational drugs are cheaper and easier for distribution in various formats such as liquid, pill, or powder $[1,8]$. Recreational drug users can have enhanced feelings of stamina and intoxicating highs and therefore be prone to sexual disinhibition by having more sexual partners or having unprotected anal intercourse (UAI) $[5,9]$.
With rapid economic development, China and other Asian countries have observed increased popularity of recreational drug use [2,10-13]. A report published in 2010 showed that $55 \%$ of Chinese illicit drug users abused recreational drugs, which exceeded the proportion of abused heroin, a traditionally abused drug in China [3]. Several studies had described the behaviors of using recreational drugs among Chinese MSM. Due to the imbalance of economic development of Chinese cities, the lifetime prevalence of using recreational club drugs varied by city: Chongqing $11.3 \%$ [14], Kunming 3.2\% [15], Shenyang 1.8\% [16], and Heilongjiang $0.3 \%$ [17]. A questionnaire survey by mail found that the 
lifetime average rate of self-reported use of club drugs in six cities was $4.6 \%$ [18]. The prevalence seems to be higher among a subgroup of Chinese male sex workers (or money boy). 12\% of money boys in Shanghai reported using ecstasy, methamphetamine, or other drugs [19], and 19.9\% in Shenzhen used Ketamine, methamphetamine, and so forth [20]. However, few studies have evaluated whether or not recreational drug using behavior was an intrinsic factor impacting the rapid increasing HIV epidemic of Chinese MSM population. It is very necessary to understand the relationship of recreational drug use and HIV infection among Chinese MSM population in order to implement targeted behavioral interventions and active treatment to control their recreational drug using behavior and HIV infection risk [4].

The primary aims of this study are to investigate the prevalence of recreational drug using behaviors, determine factors correlated with recreational drug using behavior, and evaluate the relationship between recreational drug using behavior and HIV infection among MSM in Shenyang, a large inner city in Northeast China.

\section{Methods and Materials}

2.1. Study Setting. The study was conducted in Shenyang, a capital city of Liaoning Province in northern China. The HIV epidemic has been rapidly increasing among MSM in Shenyang over the past 5-10 years; for example, the incidence rate was 4.7/100 person-years (PY) in 2007, 6.1/100 PY in 2008, and 10.2/100 PY in 2009 [21, 22].

2.2. Participant Enrollment. MSM who were living in Shenyang were recruited to participate in the study in 2011. Snowball sampling methods were used to recruit MSM participants: all MSM were invited through MSM chat rooms by a local gay community-based organization (CBO) called Shenyang Sunny Workgroup to participate in the study. Enrolled participants were asked to refer their friends and sexual partners to participate in the study.

2.3. Survey Method and Procedures. The survey was conducted at the Rainbow Harbor voluntary counseling and testing (VCT) Center, which is affiliated with the First Affiliated Hospital of China Medical University. Trained staff at the Rainbow Harbor VCT Center fully explained the study purpose, contents, and procedures at the reception room when MSM came to the clinic. Written informed consent was obtained prior to questionnaire survey. The eligibility criteria were (a) male, self-reporting anal intercourse with male partners in the past 6 months, (b) 18 years or older, and (c) able and willing to provide written informed consent.

2.4. Data Collection. Venous blood was drawn by trained nurses after a trained counselor provided pretest counseling. A structured questionnaire interview was administered faceto-face in a private counseling room. The questionnaire included sections on demographics, types, and numbers of sexual partners, condom using conditions with various sexual behaviors in the past three months, alcohol (alcohol users were defined as consuming alcohol at least six times in the past three months), illicit drug use (including recreational drug using behaviors and injection drug using behaviors), and self-perceived HIV infection risk according to each participant's sexual behaviors experience in the past three months (there are four candidate items to choose from: no risk, low risk, medium risk, and high risk).

2.5. Laboratory Testing. Sera were tested for HIV and syphilis antibodies. Serum samples were tested for HIV antibody screening using a third-generation enzyme-linked immunosorbent assay (Vironostika HIV-1/2 Microelisa System; BioMérieux, Holland). Samples with positive screening test result were confirmed by HIV-1/2 Western blot assay (HIV Blot 2.2 WB; Genelabs Diagnostics, Singapore). For syphilis testing, serum samples were screened through a rapid regain test (RPR, Diagnosis t; Shanghai Kehua, China). Specimens detected to be RPR positive were confirmed using a Treponema pallidum particle test (TPPA, Serodia, Japan). Participants who were tested positive for both TPPA and RPR were determined to be currently infected with syphilis.

2.6. Data Analysis. Data were double entered and checked using Epi-data 3.0 (The Epi Data Association Odense, Denmark, version 3.02) and analyzed using SPSS software (version 17.0; SPSS, Inc., Chicago, IL, USA). Category variables and continuous variables were described by proportions and means, respectively. Logistic regression models were used to assess the factors associated with recreational drug using behavior, such as having multiple $(\geq 3)$ male sexual partners in the past three months, and HIV infection status. Variables significant at $P<0.2$ level in the univariate analysis were entered in a multivariate stepwise logistic regression analysis model, and variables significant at $P<0.05$ level were retained in the final model.

2.7. Ethical Considerations. The questionnaire interviews were conducted in private counseling rooms. The study was anonymous, and a unique participant identification number (PID) was used for marking blood specimens and questionnaires. The testing results of HIV and syphilis infections were provided to each participant within one week. Trained staff provided individual posttest counseling. Those with positive HIV testing results were referred to the HIV/AIDS treatment clinic of the study hospital for disease surveillance, care, and support. The study protocol received ethical approval from the Institutional Review Board (IRB) of the First Affiliated Hospital of China Medical University (CMU).

\section{Results}

3.1. Characteristics of MSM Using or Not Using Recreational Drugs. A total of 640 MSM were approached for participation in this study. Ten declined and 5 were ineligible due to age $<18$ years, and the remaining 625 enrolled in the study. Of 625 participants, 145 (23.2\%) reported a history of using recreational drugs, in which 120 (19.2\%) used poppers (alkyl nitrites or rush), 32 (5.1\%) used methylmorphine phosphate 
(5.1\%), $25(4.0 \%)$ used methamphetamine, $5(0.8 \%)$ used Ketamine, 4 (0.6\%) used Ecstasy, and 3 (0.5\%) used MaGu. Two MSM participants $(0.3 \%)$ have ever injected drugs. Sixty (9.6\%) were HIV positive and 65 (10.4\%) were syphilis positive.

Table 1 showed the comparisons of demographic and behavioral characteristics between recreational drug users and nonusers. Compared with nonusers, recreational drug users were more likely to be younger and unmarried, have unprotected anal intercourse (UAI) with regular male sexual partners and multiple $(\geq 3)$ male sexual partners in the past three months $(P<0.05)$, and seek sexual partners via the Internet. Recreational drug users were less likely to be engaged in UAI with casual male sexual partners in the past 3 months (each $P<0.05$ ). There were no statistical differences in current residence, ethnic composition, education level, average monthly income, preferred role in anal sex, commercial sex, and condom use in commercial sex between two groups $(P>0.05)$.

3.2. Factors Associated with Use of Recreational Drugs. Table 2 shows the factors associated with use of recreational drugs in univariate and multivariate logistic regression analyses. In univariate analyses, age, marital status, seeking male sex partners via Internet, residence in Liaoning Province, and preferred role in anal sex were significantly associated with use of recreational drug using behavior $(P<0.2)$ and entered to multivariate analysis model. Three factors remained significant in multivariate analysis $(P<0.05)$, and adjusted odds ratio (aOR) and 95\% confidence interval (CI) were age $\leq 25$ versus (vs.) $>25$ years (1.6, 1.1-2.9), single versus married with female or cohabited with males $(2.1,1.2-$ 3.6), and seeking male sex partners via bar/dance halls versus Internet $(1.8,1.8-2.8)$.

3.3. Factors Associated with Having Multiple Male Sexual Partners. A total of 264 (42.2\%) participants reported having multiple male sexual partners $(\geq 3)$ in the past three months. Table 3 shows the factors correlated with having multiple male sexual partners by univariate and multivariate logistic regression analyses. Educational level, main route of seeking male sexual partners, preferred role in anal intercourse, and ever using recreational drugs were significantly correlated with having multiple male sexual partners (each $P<0.2$ ) in univariate analyses. All of these variables were significant in multivariate analysis $(P<0.05)$, namely, educational level of high school and below (versus college or above: $2.0,1.4-2.9$ ), seeking male sexual partners mainly through Internet (versus mainly through park/public bath: $2.0,1.1-3.8$ ), predominantly practicing receptive anal intercourse (versus insertive intercourse: $2.1,1.3-3.4$ ), practicing both insertive and receptive intercourse (versus insertive intercourse: $2.3,1.5-3.4$ ), and ever using recreational drugs (1.6, 1.1-2.3) (Table 3).

3.4. Relationship between Use of Recreational Drugs and HIV Infection. In univariate analysis, the following factors were associated with HIV infection $(P<0.05)$ : ever selling sex to male partners, self-perceived high or medium level of
HIV risk, no consistent use of condoms with causal male sexual partners, preferring receptive anal intercourse (versus insertive), ever using recreational drugs, and syphilis infection. These variables were included in multivariate analysis. The following variables were retained in the final multivariate logistic regression model as independent predictors for HIV infection: ever selling sex to male sexual partners $(3.1,1.1-$ 9.9), no consistent use of condoms with casual male sexual partners $(2.8,1.4-5.7)$, ever using recreational drugs $(3.5,2.0-$ 6.2), and syphilis coinfection (3.7, 1.9-7.2) (Table 4).

In univariate analysis, the odds of HIV infection among poppers using MSM were 4.1 times (95\% CI, 2.3-7.2, $P<$ 0.001 ) higher than the odds of nonpoppers using MSM participants, and the odds of HIV infection among methamphetamine using MSM participants were 1.8 times (95\% CI, 0.6-5.6, $P=0.276$ ) higher than the odds of nonmethamphetamine using MSM participants. These factors were not entered for multivariate analysis to avoid the collinearity problems with the recreational drug using behavior variable.

\section{Discussion}

Our study revealed that use of recreational drugs is prevalent among MSM in Shenyang city of China, and poppers and methamphetamine are the most commonly used drugs. Recreational drug use may increase sexual risk behavior and HIV infection $[1,4,8]$ and therefore post additional challenge for HIV intervention programs among MSM. The finding also underscores the importance of drug use behavioral surveillance in this population. Public health providers from China's various VCT sites should pay more attention to the collection of recreational drug using behaviors of those clients who seek consultation and HIV testing at VCT sites, whose information may be helpful to conduct targeted behavior intervention to decrease the HIV infection risk of those clients who seek consultation and HIV testing at posttest counseling stage, especially for vulnerable MSM population.

This study expands on the already extensive literature about recreational drug use among MSM in China. The prevalence of drug use among the MSM population in Shenyang, a large northeast city with medium economic level, was higher than in other parts of China [14-17, 23], including male commercial sex workers (money boys) in Shenzhen [23] and Shanghai [19]. The prevalence is similar to that among MSM in Washington D.C. (22.6\%) [24] but less than in Sydney (80.3\%) [7]. Poppers are the most common drug in this study site, compared to Ketamine in Shenzhen [23], ecstasy in Shanghai [19], and methamphetamine in six other Chinese cities [18]. Among American MSM participants in the Multicenter AIDS Cohort Study, poppers are the most common drug (61\%), followed by methamphetamine $(23 \%)$ and cocaine (14\%) [5]. The reasons for the popularity of using popper may include low cost and easy accessibility, as it is widely sold by the Internet retailers in China.

Recreational drug use was associated with younger age, unmarried status, and seeking male sexual partners through the Internet. It was suggested that this subgroup of MSM should be considered a priority targeted group for drug 
TABLE 1: Characteristics of MSM who used or did not use recreational drugs in Shenyang of China.

\begin{tabular}{|c|c|c|c|c|c|c|}
\hline \multirow{2}{*}{ Characteristics } & \multicolumn{2}{|c|}{ Drug users $(N=145)$} & \multicolumn{2}{|c|}{ Nonusers $(N=480)$} & \multirow{2}{*}{$\chi^{2}$} & \multirow{2}{*}{$P$ value } \\
\hline & Number & Proportion (\%) & Number & Proportion (\%) & & \\
\hline \multicolumn{7}{|l|}{ Age, year } \\
\hline$>25$ & 71 & 49.0 & 314 & 65.4 & 12.741 & $<0.001$ \\
\hline$\leq 25$ & 74 & 51.0 & 166 & 34.6 & & \\
\hline \multicolumn{7}{|l|}{ Residence in Liaoning Province } \\
\hline Yes & 101 & 69.7 & 367 & 76.5 & & \\
\hline No & 44 & 30.3 & 113 & 23.5 & 2.740 & 0.098 \\
\hline \multicolumn{7}{|l|}{ Ethnic } \\
\hline Han & 126 & 86.9 & 399 & 83.1 & & \\
\hline Non-Han & 19 & 13.1 & 81 & 16.9 & 1.179 & 0.278 \\
\hline \multicolumn{7}{|l|}{ Educational level } \\
\hline College and above & 59 & 40.7 & 176 & 36.7 & & \\
\hline Senior high school and below & 86 & 59.3 & 304 & 63.3 & 0.768 & 0.381 \\
\hline \multicolumn{7}{|l|}{ Marital status } \\
\hline Married with females/cohabitated with males & 19 & 13.1 & 136 & 28.3 & & \\
\hline Single & 126 & 86.9 & 344 & 71.7 & 13.850 & $<0.001$ \\
\hline \multicolumn{7}{|l|}{ Average monthly income, USD } \\
\hline$\leq 500$ & 101 & 69.7 & 351 & 73.1 & & \\
\hline$>500$ & 44 & 30.3 & 129 & 26.9 & 0.670 & 0.413 \\
\hline \multicolumn{7}{|l|}{ Ever sold sex to male partners } \\
\hline No & 139 & 95.9 & 466 & 97.1 & & \\
\hline Yes & 6 & 4.1 & 14 & 2.9 & 0.536 & 0.464 \\
\hline \multicolumn{7}{|l|}{ Main venue to seek male sex partners } \\
\hline Internet & 98 & 67.6 & 240 & 50.0 & 13.904 & 0.001 \\
\hline Bar/dance halls & 8 & 5.5 & 44 & 9.2 & & \\
\hline Park/public bath & 39 & 26.9 & 196 & 40.8 & & \\
\hline \multicolumn{7}{|l|}{ Main sexual role with males } \\
\hline Receptive anal intercourse & 29 & 20.0 & 93 & 19.4 & & \\
\hline Receptive/insertive anal intercourse & 82 & 56.6 & 240 & 50.0 & & \\
\hline Insertive anal intercourse & 34 & 23.4 & 147 & 30.6 & 2.931 & 0.231 \\
\hline \multicolumn{7}{|l|}{ Ever injecting illicit drug } \\
\hline No & 145 & 100.0 & 478 & 99.6 & & \\
\hline Yes & 0 & 0.0 & 2 & 0.4 & 0.606 & 0.436 \\
\hline \multicolumn{7}{|l|}{ UAI with regular male partners } \\
\hline No & 103 & 71.0 & 381 & 79.4 & & \\
\hline Yes & 42 & 29.0 & 99 & 20.6 & 4.434 & 0.035 \\
\hline \multicolumn{7}{|l|}{ UAI with casual male partners } \\
\hline No & 111 & 76.6 & 324 & 67.5 & & \\
\hline Yes & 34 & 23.4 & 156 & 32.5 & 4.312 & 0.038 \\
\hline \multicolumn{7}{|l|}{ UAI with commercial partners in the recent 3 months } \\
\hline No & 137 & 95.1 & 454 & 95.2 & & \\
\hline Yes & 7 & 4.9 & 23 & 4.8 & 0.000 & 0.985 \\
\hline \multicolumn{7}{|l|}{ Number of male partners in the past 3 months } \\
\hline$<3$ & 72 & 49.7 & 289 & 60.2 & & \\
\hline$\geq 3$ & 73 & 50.3 & 191 & 39.8 & 5.083 & 0.024 \\
\hline
\end{tabular}

UAI: unprotected anal intercourse. 
TABLE 2: Factors associated with using recreational drugs among MSM in Shenyang of China $(n=625)$.

\begin{tabular}{|c|c|c|c|c|c|c|}
\hline \multirow{2}{*}{ Factors } & \multirow{2}{*}{$N(\%)$} & \multirow{2}{*}{ Number of recreational drug use (\%) } & \multicolumn{2}{|c|}{ Univariate analysis } & \multicolumn{2}{|c|}{ Multivariate analysis } \\
\hline & & & OR $(95 \% \mathrm{CI})$ & $P$ & $\mathrm{aOR}(95 \% \mathrm{CI})$ & $P$ value \\
\hline \multicolumn{7}{|l|}{ Age, year } \\
\hline$>25$ & $385(61.6)$ & $71(18.4)$ & 1.0 & & & \\
\hline$\leq 25$ & $240(38.4)$ & $74(30.8)$ & $2.0(1.4-2.9)$ & $<0.001$ & $1.6(1.1-2.9)$ & 0.017 \\
\hline \multicolumn{7}{|l|}{ Residence in Liaoning Province } \\
\hline Yes & $157(25.1)$ & $44(28.0)$ & 1.0 & & & \\
\hline No & $468(74.9)$ & $101(21.6)$ & $0.7(0.5-1.1)$ & 0.099 & & \\
\hline \multicolumn{7}{|l|}{ Marital status } \\
\hline $\begin{array}{l}\text { Married with females/cohabitated } \\
\text { with males }\end{array}$ & $155(24.8)$ & $19(12.3)$ & 1.0 & & & \\
\hline Single & $470(75.2)$ & $126(26.8)$ & $2.6(1.6-4.4)$ & $<0.001$ & $2.1(1.2-3.6)$ & 0.008 \\
\hline \multicolumn{7}{|l|}{ Educational level } \\
\hline College and above & $390(62.4)$ & $86(22.1)$ & 1.0 & & & \\
\hline Senior high school and below & $235(37.6)$ & $59(25.1)$ & $1.2(0.8-1.7)$ & 0.381 & & \\
\hline \multicolumn{7}{|l|}{ Main venue to seek male sex partners } \\
\hline Park/public bath & $235(37.6)$ & $39(16.6)$ & 1.0 & & & \\
\hline Internet & $338(54.1)$ & $98(29.0)$ & $2.1(1.4-3.1)$ & 0.001 & $1.8(1.2-2.8)$ & 0.005 \\
\hline Bar/dance halls & $52(8.3)$ & $8(15.4)$ & $0.9(0.4-2.1)$ & 0.914 & $1.0(0.4-2.2)$ & 0.968 \\
\hline \multicolumn{7}{|l|}{ Average month income (USD) } \\
\hline$\leq 500$ & $452(72.3)$ & $101(22.3)$ & 1.0 & & & \\
\hline$>500$ & $173(27.7)$ & $44(25.4)$ & $1.2(0.8-1.8)$ & 0.413 & & \\
\hline \multicolumn{7}{|l|}{ Main sexual role with males } \\
\hline Receptive anal intercourse & $181(29.0)$ & $34(18.8)$ & 1.0 & & & \\
\hline Receptive/insertive anal intercourse & $122(19.5)$ & $29(23.8)$ & $1.3(0.8-2.4)$ & 0.295 & & \\
\hline Insertive anal intercourse & $322(51.5)$ & $82(25.5)$ & $1.50(0.9-2.3)$ & 0.089 & & \\
\hline
\end{tabular}

use surveillance and intervention. This study also confirmed that the Internet is the major means of seeking male sexual partners for Chinese MSM $[15,16,25]$, and this group is also more likely to use recreational drugs.

Recreational drug using behavior was positively correlated with HIV infection among studied MSM participants, which was in accordance with previous findings among MSM populations in developed countries [5-7, 24, 26]. Recreational drug using MSM had significantly more numbers of multiple male sexual partners in this study, which partly explained their higher HIV infection risk. Previous studies showed that poppers could relax rectal muscles and reduce pain during sex, and therefore use of poppers was associated with increased duration of anal intercourse [1], which can further increase the risk of acquiring HIV. Our study found that poppers using MSM had a 4.1 times greater risk of acquiring HIV than nonusers, which is similar to the finding in the prospective cohort study among American MSM that poppers using MSM had 2.1 times risk of HIV seroconversion [5]. Our study also showed that methamphetamine using MSM had 1.8 times more risk to acquire HIV than nonusers, which was similar to the 2.5 times HIV risk among American MSM [5]. Due to the low prevalence of methamphetamine use, our study did not find a statistically significant relationship between methamphetamine use and HIV infection.
Syphilis can cause genital ulcer diseases (GUDs) and increase HIV infection. Our study showed that syphilis infection was associated with HIV infection, and this finding is consistent with other Chinese studies [15, 16, 21, 27]. It underscores that early testing and timely treatment of syphilis infection should be considered as a measure of HIV prevention.

Our study also confirmed the finding from our previous study that UAI with casual male sexual partners was a risk factor for HIV infection [16]. Although HIV serosorting is considered an effective risk reduction strategy among high risk MSM in the USA [28], this strategy may not be so beneficial for Chinese MSM, because the benefit of this strategy relies on high coverage and frequency of HIV testing [29], while in 2009 only $43.7 \%$ of Chinese MSM had ever taken an HIV testing in the past 12 months [30].

Selling sex was independently associated with high HIV risk, and these MSM who sell sex are generally called money boys. Selling sex is often correlated with recreational drug use and a high number of sexual partners $[19,23]$, both of which may increase HIV risk.

Our study has some strengths. Study participants were recruited and interviewed by trained members of a gay community-based organization that has been our long collaborating partner. We carefully phrased some sensitive 
TABLE 3: Factors correlated with having multiple male sexual partners $(\geq 3)$ among Shenyang MSM by multivariate logistic regression $(n=$ 625).

\begin{tabular}{|c|c|c|c|c|c|c|}
\hline \multirow{2}{*}{ Factors } & \multirow{2}{*}{$N(\%)$} & \multirow{2}{*}{$\begin{array}{l}\text { Number of having } \geq 3 \text { male } \\
\text { partners (proportion, } \% \text { ) }\end{array}$} & \multicolumn{2}{|c|}{ Univariate analysis } & \multicolumn{2}{|c|}{ Multivariate analysis } \\
\hline & & & OR $(95 \% \mathrm{CI})$ & $P$ value & $\mathrm{aOR}(95 \% \mathrm{CI})$ & $P$ value \\
\hline \multicolumn{7}{|l|}{ Age, year } \\
\hline$>25$ & $385(61.6)$ & $171(44.4)$ & 1.0 & & & \\
\hline$\leq 25$ & $240(38.4)$ & $93(38.8)$ & $0.8(0.6-1.1)$ & 0.163 & & \\
\hline \multicolumn{7}{|l|}{ Residence in Liaoning Province } \\
\hline Yes & $157(25.1)$ & $62(39.5)$ & 1.0 & & & \\
\hline No & $468(74.9)$ & $202(43.2)$ & $1.2(0.8-1.7)$ & 0.420 & & \\
\hline \multicolumn{7}{|l|}{ Marital status } \\
\hline $\begin{array}{l}\text { Married with females/Cohabitated } \\
\text { with males }\end{array}$ & $155(24.8)$ & $65(41.9)$ & 1.0 & & & \\
\hline Single & $470(75.2)$ & $199(42.3)$ & $1.0(0.7-1.5)$ & 0.929 & & \\
\hline \multicolumn{7}{|l|}{ Educational level } \\
\hline College and above & $235(37.6)$ & $76(32.3)$ & 1.0 & & & \\
\hline Senior high school and below & $390(62.4)$ & $188(48.2)$ & $1.9(1.4-2.7)$ & $<0.001$ & $2.0(1.4-2.9)$ & $<0.001$ \\
\hline \multicolumn{7}{|l|}{ Main venues to seek male sex partners } \\
\hline Internet & $338(54.1)$ & $139(41.1)$ & 1.0 & & & \\
\hline Bar/dance halls & $52(8.3)$ & $32(61.5)$ & $2.3(1.3-4.2)$ & 0.007 & $2.0(1.1-3.8)$ & 0.027 \\
\hline Park/public bath & $235(37.6)$ & $93(39.6)$ & $0.9(0.7-1.3)$ & 0.710 & $0.9(0.6-1.3)$ & 0.471 \\
\hline \multicolumn{7}{|l|}{ Average month income (USD) } \\
\hline$\leq 500$ & $452(72.3)$ & $189(41.8)$ & 1.0 & & & \\
\hline$>500$ & $173(27.7)$ & $75(43.4)$ & $1.1(0.7-1.5)$ & 0.728 & & \\
\hline \multicolumn{7}{|l|}{ Main sexual role with males } \\
\hline Insertive anal intercourse & $181(29.0)$ & $53(29.3)$ & 1.0 & & & \\
\hline Receptive anal intercourse & $122(19.5)$ & $54(44.3)$ & $1.9(1.2-3.1)$ & 0.008 & $2.1(1.3-3.4)$ & 0.003 \\
\hline Receptive/insertive anal intercourse & $322(51.5)$ & $157(48.8)$ & $2.3(1.6-3.4)$ & $<0.001$ & $2.3(1.5-3.4)$ & $<0.001$ \\
\hline \multicolumn{7}{|l|}{ Ever used recreational drugs } \\
\hline No & $480(76.8)$ & $191(39.8)$ & 1.0 & & & \\
\hline Yes & $145(23.2)$ & $73(50.3)$ & $1.5(1.1-2.2)$ & 0.025 & $1.6(1.1-2.3)$ & 0.027 \\
\hline
\end{tabular}

questions. For example, instead of asking "whether you once used illicit drugs," the question is phrased as "whether you once used special substances" to reduce social disability bias.

There are also some limitations. First, the nature of a cross-sectional study prevents concluding any causal relationship between risk factors and HIV infection. Second, the major exposure factor (recreational drug use) was selfreported, the assessment of exposure was weak, and underreporting of the condition was possible in settings where using illegal drugs may be severely punished. Lastly, lifetime recreational drug using behaviors may be different from recreational drug using behaviors immediately prior to having sex with male sexual partners. Research should be continually implemented to further determine the relationships between recreational drug using behaviors, unprotected anal intercourse, and HIV infection status among this vulnerable MSM population.

In summary, recreational drug use is common among Chinese MSM and is associated with having multiple male sexual partners and increased risk of HIV infection. HIV intervention programs targeting MSM should include a component for reducing recreational drug use and its detrimental effects.

\section{Conflict of Interests}

This study was supported by the Mega-Projects of National Science Research for the 12th Five-Year Plan (2012ZX10001006), China-Gates Foundation Cooperation Programme, and the National Nature Science Foundation of China (81001291). The funding organization had no role in the development of study design or in the collection, analysis, and interpretation of data. All authors declare no conflict of interests.

\section{Authors' Contribution}

Jun-Jie Xu conceived and designed the experiments. Hong Shang Performed the study and experiments. Jun-Jie $\mathrm{Xu}$, Zhen-Xing Chu, Qing-Hai $\mathrm{Hu}$, and Jing Zhang analyzed 
TABLE 4: Factors associated with HIV infection among MSM in Shenyang of China $(n=625)$.

\begin{tabular}{|c|c|c|c|c|c|c|}
\hline \multirow{2}{*}{ Factors } & \multirow{2}{*}{$N(\%)$} & \multirow{2}{*}{ Number of HIV positives (\%) } & \multicolumn{2}{|c|}{ Univariate analysis } & \multicolumn{2}{|c|}{ Multivariate analysis } \\
\hline & & & OR $(95 \% \mathrm{CI})$ & $P$ value & $\mathrm{aOR}(95 \% \mathrm{CI})$ & $P$ value \\
\hline \multicolumn{7}{|l|}{ Age, year } \\
\hline$>25$ & $385(61.6)$ & $30(7.8)$ & & & & \\
\hline$\leq 25$ & $240(38.4)$ & $30(12.5)$ & $1.7(1.0-2.9)$ & 0.054 & & \\
\hline \multicolumn{7}{|l|}{ Residence in Liaoning Province } \\
\hline Yes & $157(25.1)$ & $17(10.8)$ & & & & \\
\hline No & $468(74.9)$ & $43(9.2)$ & $0.8(0.5-1.5)$ & 0.547 & & \\
\hline \multicolumn{7}{|l|}{ Ethnics } \\
\hline Han & $100(16.0)$ & $12(12.0)$ & & & & \\
\hline Non-Han & $525(84.0)$ & $48(9.1)$ & $1.4(0.7-2.7)$ & 0.376 & & \\
\hline \multicolumn{7}{|l|}{ Educational level } \\
\hline College and above & $235(37.6)$ & $26(11.1)$ & & & & \\
\hline Senior high school and below & $390(62.4)$ & $34(8.7)$ & $0.8(0.4-1.3)$ & 0.336 & & \\
\hline \multicolumn{7}{|l|}{ Marital status } \\
\hline $\begin{array}{l}\text { Married with females/cohabitated } \\
\text { with males }\end{array}$ & $155(24.8)$ & $14(9.0)$ & & & & \\
\hline Single & $470(75.2)$ & $46(9.8)$ & $1.1(0.6-2.0)$ & 0.782 & & \\
\hline \multicolumn{7}{|l|}{ Average month income (USD) } \\
\hline$\leq 500$ & $452(72.3)$ & $37(8.2)$ & & & & \\
\hline$>500$ & $173(27.7)$ & $23(13.3)$ & $1.7(1.0-3.0)$ & 0.055 & & \\
\hline \multicolumn{7}{|l|}{ Ever selling sex to male partners } \\
\hline No & $605(96.8)$ & $55(9.1)$ & & & & \\
\hline Yes & $20(3.2)$ & $5(25.0)$ & $3.3(1.2-9.5)$ & 0.025 & $3.1(1.0-9.9)$ & 0.05 \\
\hline \multicolumn{7}{|c|}{ Main venue for seeking male sex partners } \\
\hline Internet & $235(37.6)$ & $23(9.8)$ & & & & \\
\hline Bar/dance halls & $338(54.1)$ & $33(9.8)$ & $1.0(0.6-1.7)$ & 0.675 & & \\
\hline Park/public bath & $52(8.3)$ & $4(7.7)$ & $0.8(0.3-2.3)$ & 0.640 & & \\
\hline \multicolumn{7}{|l|}{ Self-perceived HIV infection risk } \\
\hline No risk & $169(27.1)$ & $9(5.3)$ & & & & \\
\hline Low risk & $264(42.4)$ & $27(10.2)$ & $2.0(0.9-4.4)$ & 0.076 & & \\
\hline Medium risk & $116(18.6)$ & $14(12.1)$ & $2.4(1.0-5.8)$ & 0.045 & & \\
\hline High risk & $36(5.8)$ & $7(19.4)$ & $4.3(1.5-12.4)$ & 0.007 & & \\
\hline \multicolumn{7}{|l|}{$\begin{array}{l}\text { Condom using with casual male partners } \\
\text { in the past } 3 \text { months }\end{array}$} \\
\hline Consistently used & $439(70.2)$ & $39(8.9)$ & & & & \\
\hline Seldom used & $116(18.6)$ & $7(6.0)$ & $0.7(0.3-1.5)$ & 0.325 & $0.8(0.3-1.9)$ & 0.601 \\
\hline Never used & $70(11.2)$ & $14(20.0)$ & $2.6(1.3-5.0)$ & 0.004 & $2.8(1.4-5.7)$ & $<0.001$ \\
\hline \multicolumn{7}{|l|}{ Preferred role in anal intercourse } \\
\hline Mainly insertive & $181(29.0)$ & $11(6.1)$ & & & & \\
\hline Mainly receptive & $122(19.5)$ & $16(13.1)$ & $2.3(1.0-5.2)$ & 0.039 & & \\
\hline Receptive/insertive anal intercourse & $322(51.5)$ & $33(10.2)$ & $1.8(0.9-3.6)$ & 0.116 & & \\
\hline \multicolumn{7}{|l|}{$\begin{array}{l}\text { Number of male sexual partners in the } \\
\text { past } 3 \text { months }\end{array}$} \\
\hline$<3$ & $361(57.8)$ & $38(10.5)$ & & & & \\
\hline$\geq 3$ & $264(42.2)$ & $22(8.3)$ & $0.8(0.4-1.3)$ & 0.359 & & \\
\hline \multicolumn{7}{|l|}{ Ever using recreational drugs } \\
\hline No & $480(76.8)$ & $31(6.5)$ & & & & \\
\hline Yes & $145(23.2)$ & $29(20.0)$ & $3.6(2.1-6.3)$ & $<0.001$ & $3.5(2.0-6.2)$ & $<0.001$ \\
\hline Alcohol using behavior & & & & & & \\
\hline No & $181(57.3)$ & $37(20.4)$ & & & & \\
\hline Yes & $135(42.7)$ & $20(14.8)$ & $0.6(0.3-1.6)$ & 0.649 & & \\
\hline Reported male circumcision status & & & & & & \\
\hline No & $581(93.0)$ & $54(9.3)$ & & & & \\
\hline Yes & $44(7.0)$ & $6(13.6)$ & $1.5(0.6-3.8)$ & 0.349 & & \\
\hline Syphilis infection & & & & & & \\
\hline No & $560(89.6)$ & $43(7.7)$ & & & & \\
\hline Yes & $65(10.4)$ & $17(26.2)$ & $4.3(2.3-8.0)$ & $<0.001$ & $3.7(1.9-7.2)$ & $<0.001$ \\
\hline
\end{tabular}


the data. Jun-Jie $\mathrm{Xu}$ and Zhen-Xing Chu contributed to reagents/materials/analysis tools. Jun-Jie Xu,Han-Zhu Qian, Yong-Jun Jiang, Wen-Qing Geng and Christiana Meng Zhang Wrote and revised the paper. All authors read and approved the final paper.

\section{Acknowledgments}

The authors thank all the MSM who participated in the study. Finally, we thank all the staff from Shenyang sunny volunteer group for helping to recruit MSM participants and conduct related questionnaire surveys.

\section{References}

[1] L. N. Drumright, T. L. Patterson, and S. A. Strathdee, "Club drugs as causal risk factors for HIV acquisition among men who have sex with men: a review," Substance Use \& Misuse, vol. 41, no. 10-12, pp. 1551-1601, 2006.

[2] Y. Y. Ding, N. He, and R. Detels, "Circumstances of initiation into new-type drug use among adults in Shanghai: are there differences by types of first new-type drug used?" Drug and Alcohol Dependence, vol. 131, pp. 278-283, 2013.

[3] Y. Ding and N. He, "Club drugs and HIV/STI infection: a new public health concern in China," Fudan University Journal of Medical Sciences, vol. 39, pp. 551-556, 2012.

[4] G. Colfax, G.-M. Santos, P. Chu et al., "Amphetamine-group substances and HIV," The Lancet, vol. 376, no. 9739, pp. 458474, 2010.

[5] M. W. Plankey, D. G. Ostrow, R. Stall et al., "The relationship between methamphetamine and popper use and risk of HIV seroconversion in the multicenter AIDS cohort study," Journal of Acquired Immune Deficiency Syndromes, vol. 45, no. 1, pp. 8592, 2007.

[6] C. P. Bonell, F. C. I. Hickson, P. Weatherburn, and D. S. Reid, "Methamphetamine use among gay men across the UK," International Journal of Drug Policy, vol. 21, no. 3, pp. 244-246, 2010.

[7] G. Prestage, L. Degenhardt, F. Jin et al., "Predictors of frequent use of amphetamine type stimulants among HIV-negative gay men in Sydney, Australia," Drug and Alcohol Dependence, vol. 91, no. 2-3, pp. 260-268, 2007.

[8] K. H. Mayer, G. Colfax, and R. Guzman, "Club drugs and HIV infection: a review," Clinical Infectious Diseases, vol. 42, no. 10, pp. 1463-1469, 2006.

[9] L. N. Drumright, S. J. Little, S. A. Strathdee et al., "Unprotected anal intercourse and substance use among men who have sex with men with recent HIV infection," Journal of Acquired Immune Deficiency Syndromes, vol. 43, no. 3, pp. 344-350, 2006.

[10] K. A. J. Laidler, "The rise of club drugs in a heroin society: the case of Hong Kong," Substance Use \& Misuse, vol. 40, no. 9-10, pp. 1257-1278, 2005.

[11] K. Kulsudjarit, "Drug problem in southeast and southwest Asia," Annals of the New York Academy of Sciences, vol. 1025, pp. 446457, 2004.

[12] S. G. Sherman, D. German, B. Sirirojn, N. Thompson, A. Aramrattana, and D. D. Celentano, "Initiation of methamphetamine use among young Thai drug users: a qualitative study," Journal of Adolescent Health, vol. 42, no. 1, pp. 36-42, 2008.

[13] P. I. Dargan and D. M. Wood, "Recreational drug use in the Asia Pacific Region: improvement in our understanding of the problem through the UNODC Programmes," Journal of Medical Toxicology, vol. 8, pp. 295-299, 2012.

[14] X.-B. Ding, L.-G. Feng, J. Xu et al., "Study on the prevalence of HIV, Syphilis, HCV and HSV -II and its associated factors among 743 men who have sex with men in Chongqing," Chinese Journal of Disease Control \& Prevention, vol. 14, pp. 227-231, 2010.

[15] J. Xu, M. An, X. Han et al., "Prospective cohort study of HIV incidence and molecular characteristics of HIV among men who have sex with men (MSM) in Yunnan Province, China," BMC Infectious Diseases, vol. 13, article 3, 2013.

[16] J.-J. Xu, K. H. Reilly, C.-M. Lu et al., "A cross-sectional study of HIV and syphilis infections among male students who have sex with men (MSM) in northeast China: implications for implementing HIV screening and intervention programs," BMC Public Health, vol. 11, article 287, 2011.

[17] Y. Li, K. Wang, X. Dong et al., "The analysis of surveillance report of HIV high risk populations of Heilongjiang Province in 2010," Chinese Primary Health Care, vol. 25, pp. 59-61, 2011.

[18] X.-F. Li, B.-C. Zhang, Z.-Z. Yu et al., "A survey on condom use and related behaviors among 1295 men who have sex with men in six cities of China," Chinese Journal of AIDS \& STD, vol. 16, pp. 449-452, 2010.

[19] N. He, F. Y. Wong, Z. J. Huang et al., "HIV risks among two types of male migrants in Shanghai, China: money boys vs. general male migrants," AIDS, vol. 21, no. 8, pp. S73-S79, 2007.

[20] S. Liu and R. Detels, "Recreational drug use: an emerging concern among venue-based male sex workers in China," Sexually Transmitted Diseases, vol. 39, no. 4, pp. 251-252, 2012.

[21] J.-J. Xu, M. Zhang, K. Brown et al., "Syphilis and HIV seroconversion among a 12-month prospective cohort of men who have sex with men in Shenyang, China," Sexually Transmitted Diseases, vol. 37, no. 7, pp. 432-439, 2010.

[22] M. Zhang, Z. X. Chu, H. L. Wang, J. Xu, C. Lu, and H. Shang, "A rapidly increasing incidence of HIV and syphilis among men who have sex with men in a major city of China," AIDS Research and Human Retroviruses, vol. 27, no. 11, pp. 1139-1140, 2011.

[23] G. Liu, W. Cai, L. Chen et al., "Study on influential factors and epidemiological characteristics of drug abuse among men who have sex with men in Shenzhen," Chinese Journal Of Disease Control \& Prevention, vol. 14, pp. 1063-11065, 2010.

[24] Y. Li, J. J. Baker, V. R. Korostyshevskiy, R. S. Slack, and M. W. Plankey, "The Association of Intimate Partner Violence, Recreational Drug Use with HIV Seroprevalence among MSM," AIDS and Behavior, vol. 16, pp. 491-498, 2012.

[25] H. Zou, Z. Wu, J. Yu et al., "Sexual risk behaviors and HIV infection among men who have sex with men who use the internet in Beijing and Urumqi, China," Journal of Acquired Immune Deficiency Syndromes, vol. 53, no. 1, pp. S81-S87, 2010.

[26] D. G. Ostrow, M. W. Plankey, C. Cox et al., "Specific sex drug combinations contribute to the majority of recent HIV seroconversions among MSM in the MACS," Journal of Acquired Immune Deficiency Syndromes, vol. 51, no. 3, pp. 349-355, 2009.

[27] Y. Ruan, D. Li, X. Li et al., "Relationship between syphilis and HIV infections among men who have sex with men in Beijing, China," Sexually Transmitted Diseases, vol. 34, no. 8, pp. 592597, 2007.

[28] S. Cassels, T. W. Menza, S. M. Goodreau, and M. R. Golden, "HIV serosorting as a harm reduction strategy: evidence from Seattle, Washington," AIDS, vol. 23, no. 18, pp. 2497-2506, 2009. 
[29] L. M. McDaid and G. J. Hart, "Serosorting and strategic positioning during unprotected anal intercourse: are risk reduction strategies being employed by gay and bisexual men in Scotland?" Sexually Transmitted Diseases, vol. 39, pp. 735-738, 2012.

[30] E. P. F. Chow, D. P. Wilson, and L. Zhang, "The rate of HIV testing is increasing among men who have sex with men in China," HIV Medicine, vol. 13, no. 5, pp. 255-263, 2012. 


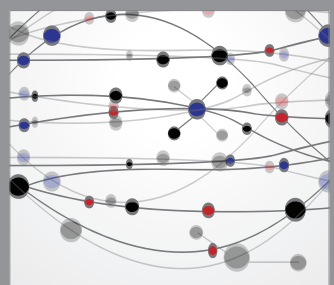

The Scientific World Journal
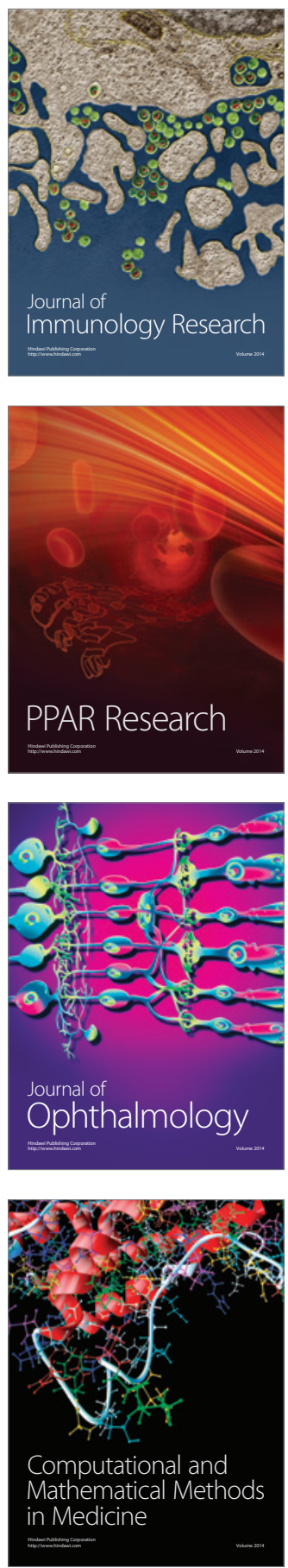

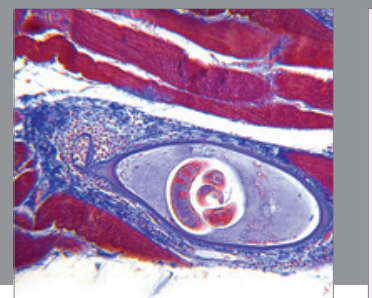

Gastroenterology

Research and Practice
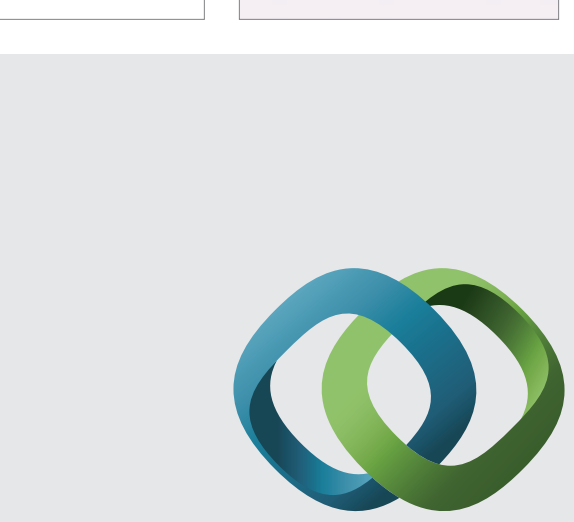

\section{Hindawi}

Submit your manuscripts at

http://www.hindawi.com
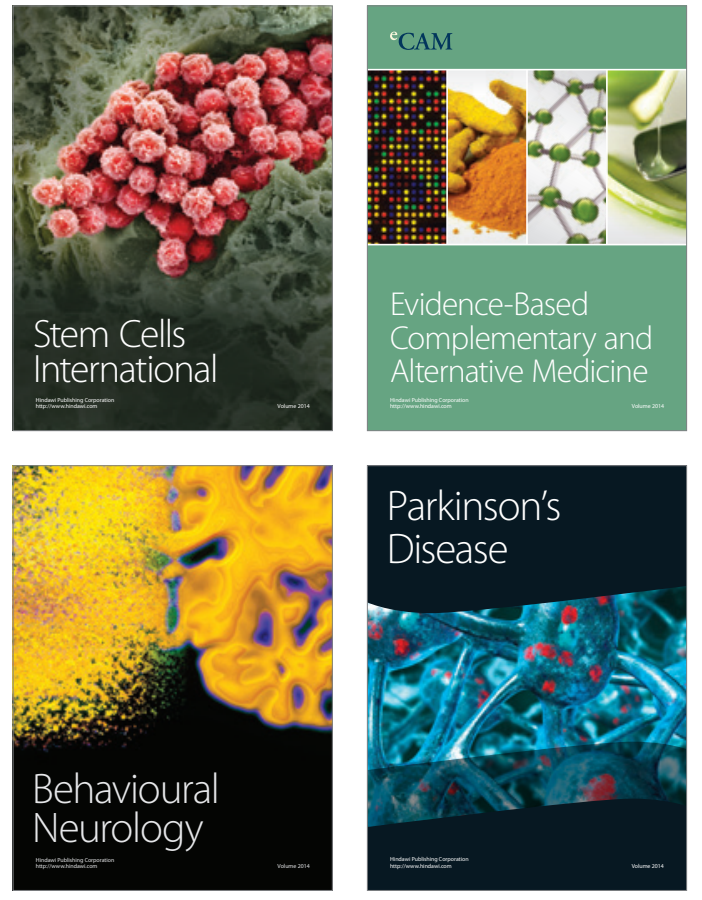
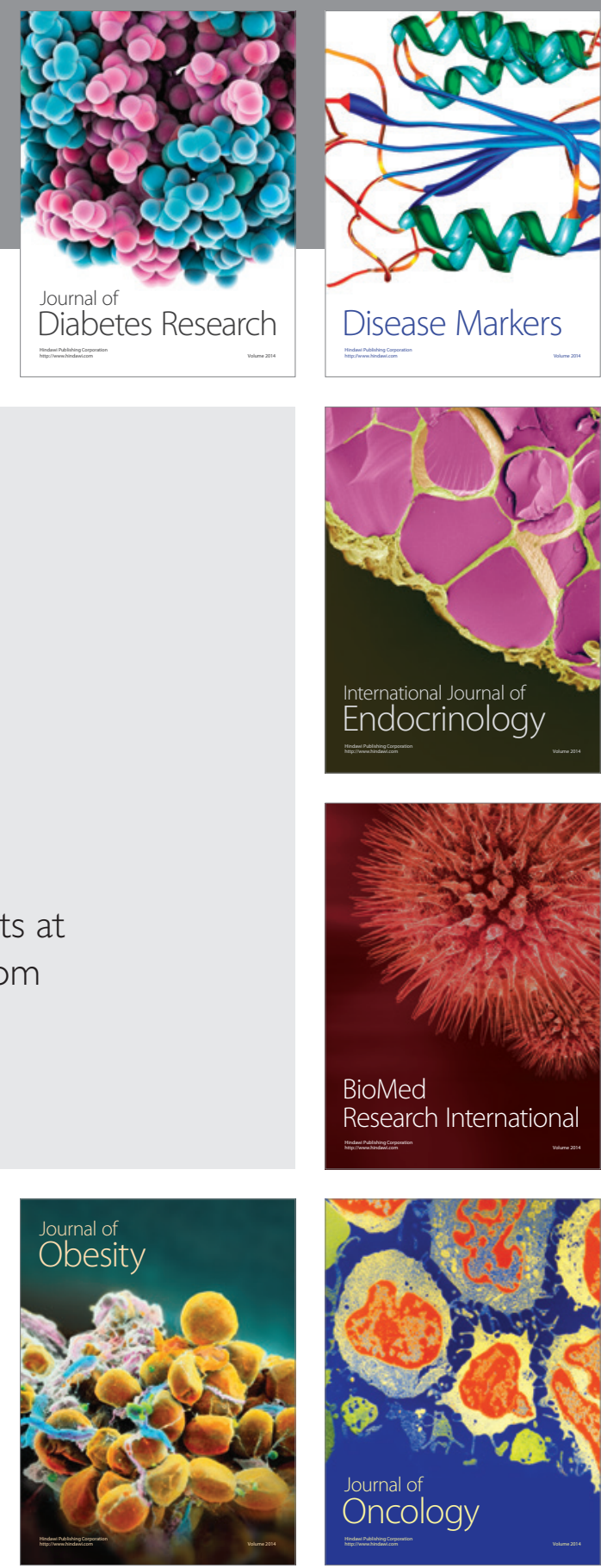

Disease Markers
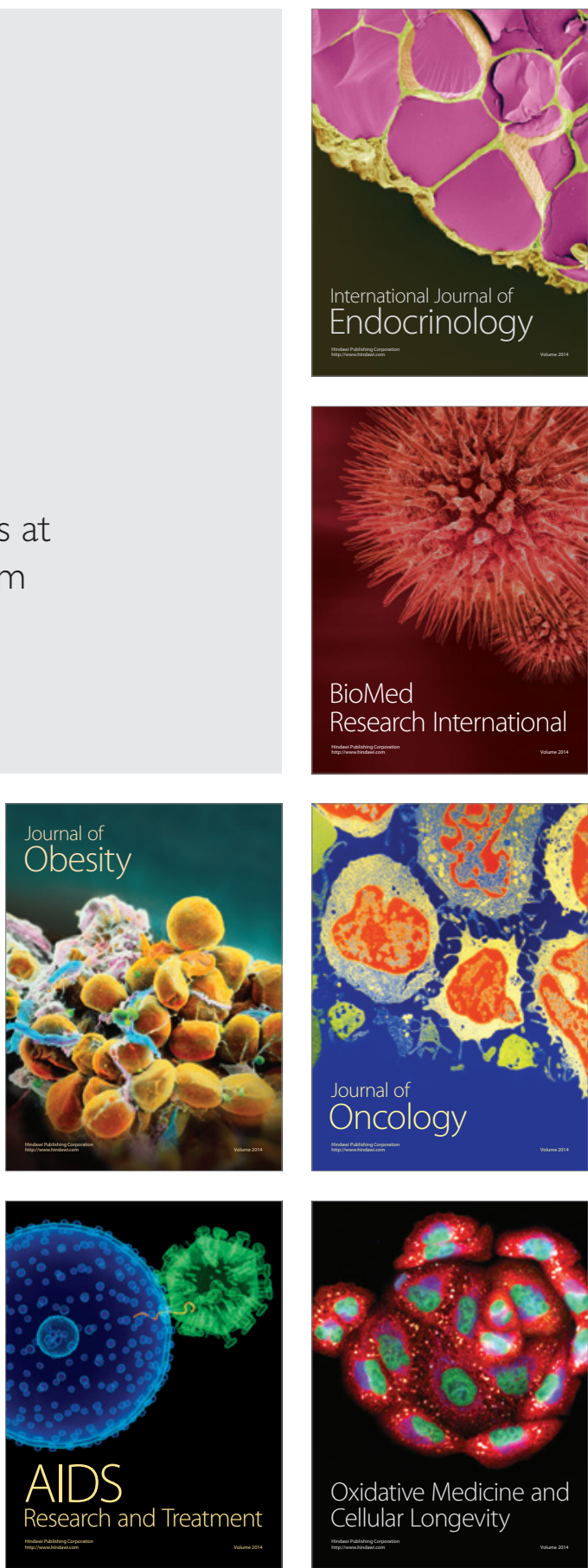